\title{
Percutaneous epididymal sperm aspiration as a method for sperm retrieval in men with obstructive azoospermia seeking fertility: operative and laboratory aspects
}

\author{
Sandro C. Esteves ${ }^{1}$ \\ ${ }^{1}$ ANDROFERT - Andrology and Human Reproduction Clinic, Campinas, SP, Brasil
}

\section{ABSTRACT}

Introduction: Congenital bilateral absence of vas deferens (CBAVD) is a non-treatable cause of obstructive azoospermia (OA). However, the affected men can father children by undergoing sperm retrieval (SR) and intracytoplasmic sperm injection (ICSI).

Materials and Methods: This video describes percutaneous epididymal sperm aspiration (PESA), performed on a 36 year-old male with CBAVD. In PESA the goal is to obtain epididymal fluid. A hypodermic needle attached to a 1 cc syringe is inserted through the skin into the corpus or caput epididymis. Gentle negative pressure is applied to aspirate the epididymal fluid, which is sent to the laboratory for examination.

Results: Total number of spermatozoa retrieved after a single puncture was 3.5 million sperm, of which $29 \%$ were motile. Motile spermatozoa with normal morphology were selected and injected into the oocyte cytoplasm, while excess retrieved sperm were cryopreserved. The operative time was 10 minutes. The patient recovered his normal activities within the next day and no complications were recorded. In a series involving 32 men with CBAVD, success rate at obtaining motile sperm by PESA was 96.8\%, with a complication rate of 3.1\%. ICSI carried out with spermatozoa retrieved by PESA resulted in a live birth rate of $34.4 \%$ per attempt. The short-term outcome of resulting offspring was comparable with those obtained in other categories of OA.

Conclusion: PESA is a simple, quick, and successful procedure to retrieve sperm from men with OA due to CBAVD. Retrieved sperm can be successfully used to generate healthy offspring with the aid of ICSI.

\section{ARTICLE INFO}

ARTICLE INFO Available at: www.brazjurol.com.br/videos/july_august_2015/Esteves_817_818video.htm

Int Braz J Urol. 2015; 41 (Video \#6): 817-8

Submitted for publication:

February 04, 2015

Accepted after revision:

July 12, 2015
Correspondence address: Sandro C. Esteves, MD, PhD ANDROFERT Andrology and Human Reproduction Clinic, Av. Dr. Heitor Penteado 1464 Campinas, São Paulo 13075-460, Brazil E-mail: s.esteves@androfert.com.br 


\section{EDITORIAL COMMENT}

In this very nice video, the authors highlight the clinical merit of percutaneous epididymal sperm aspiration (PESA) in the management of obstructive azoospermia. The video highlights not only the key features of this technique but depict within a consecutive cohort of 32 male patients that excellent outcomes can be achieved, with a very acceptable low complication rate. It clearly validates PESA as a reliable and excellent choice in the management of obstructive azoospermia. There are clearly many factors which must be considered in deciding which therapeutic intervention is selected in the management of obstructive

\section{EDITORIAL COMMENT}

Sperm can be obtained by microepididymal sperm aspiration (MESA), percutaneous sperm aspiration (PESA), and testicular sperm extraction (TESE), from patients with congenital absence of the vas deferens (CBAVD). The authors of the video on PESA as a method for sperm retrieval in obstructive azoospermia nicely demonstrate this technique in men with CBAVD. These patients have an abnormal epididymis from this condition making the retrieval more challenging (1). In their case series of 32 men with CBAVD, success rate at obtaining motile sperm by PESA was $96.8 \%$, with a live birth rate of $34.4 \%$ per attempt. The short-term outcome of resulting offspring was azoospermia such as the success in achieving high concentrations of motile sperm while imparting minimal to no risk. Clearly, PESA achieves these endpoints quite successfully and hence constitutes an excellent choice in fertility management provided intracytoplasmic sperm injection is readily available to couples thereafter and hopefully resulting in a successful conception.

Philippe E. Spiess, M.D. Video Section Editor, International Brazilian Journal of Urology E-mail:Philippe.Spiess@moffitt.org

comparable with those obtained in other types of obstructive azoospermia. Percutaneous aspiration of sperm is an effective, safe, and reproducible means of obtaining adequate sperm in men who have obstructive azoospermia due to CBAVD.

\section{Reference}

1. Dohle GR, Ramos L, Pieters MH, Braat DD, Weber RF. Surgical sperm retrieval and intracytoplasmic sperm injection as treatment of obstructive azoospermia. Hum Reprod. 1998;13:620-3.

Tariq Hakki, MD Advanced Urology Male Reproductive Medicine and Surgery 1557 Janmar Road Snellville, GA 30097 E-mail:tariq7@gmail.com 